\title{
Role of the circadian system in cardiovascular disease
}

\author{
Saurabh S. Thosar, Matthew P. Butler, and Steven A. Shea \\ Oregon Institute of Occupational Health Sciences, Oregon Health and Science University, Portland, Oregon, USA.
}

\begin{abstract}
All species organize behaviors to optimally match daily changes in the environment, leading to pronounced activity/rest cycles that track the light/dark cycle. Endogenous, approximately 24-hour circadian rhythms in the brain, autonomic nervous system, heart, and vasculature prepare the cardiovascular system for optimal function during these anticipated behavioral cycles. Cardiovascular circadian rhythms, however, may be a double-edged sword. The normal amplified responses in the morning may aid the transition from sleep to activity, but such exaggerated responses are potentially perilous in individuals susceptible to adverse cardiovascular events. Indeed, the occurrence of stroke, myocardial infarction, and sudden cardiac death all have daily patterns, striking most frequently in the morning. Furthermore, chronic disruptions of the circadian clock, as with night-shift work, contribute to increased cardiovascular risk. Here we highlight the importance of the circadian system to normal cardiovascular function and to cardiovascular disease, and identify opportunities for optimizing timing of medications in cardiovascular disease.
\end{abstract}

\section{Introduction}

Almost all cardiovascular (CV) variables that have been assessed at different times of day and night in humans have been found to have a day/night pattern, including blood pressure (BP) (1-3), heart rate (HR) (3), circulating catecholamines (4), blood coagulation markers (5), and vascular endothelial function (6). Moreover, epidemiological data reveal a robust morning increase in adverse CV events, including stroke $(7,8)$, myocardial infarction (9-11), serious ventricular arrhythmias (12), and sudden cardiac death (refs. 11, 13, and Figure 1). Typical morning behaviors (e.g., arousal from sleep, sudden change in posture after overnight recumbency, increased activity, and psychological stress) cause increases in sympathetic tone, vasoconstriction, peripheral arterial resistance, $\mathrm{HR}, \mathrm{BP}$, and platelet aggregability, and decreases in thrombolytic activity, parasympathetic tone, and vascular endothelial function (14). These daily $\mathrm{CV}$ fluctuations arise from the negative-feedback reactions to daily changes in the environment or behaviors that summate with anticipatory feed-forward effects on the CV system from the endogenous circadian system (Figure 2). Such CV changes in the morning may be advantageous in healthy individuals, but could be implicated in precipitating an adverse CV event in susceptible individuals (14-17).

Because of the Earth's rotation, life on earth has evolved under conditions of predictable daily cycles of light and darkness. Organisms arrange their daily patterns of behaviors, such as the 24-hour fasting/feeding and rest/activity cycles, to optimally match these predictable changes in the environment. An endogenous circadian clock prepares the body for these anticipated daily cycles. For example, circulating cortisol and core body temperature increase during the last part of the night to prepare the metabolic and circulatory systems for abrupt increases in energy expenditure and activity soon after awakening (18). These anticipatory changes imply that physiological reactions are suboptimal without the circa-

Conflict of interest: The authors have declared that no conflict of interest exists. Reference information: J Clin Invest. 2018;128(6):2157-2167. https://doi.org/10.1172/JCl80590. dian system appropriately priming or dampening responses based on time of day or night, perhaps because delays exist in some negative-feedback control systems (e.g., maximal release of cortisol after an acute stress can take up to 10 minutes; ref. 19).

We review the organization of the circadian system and discuss its role in the heart, vasculature, and blood; summarize circadian rhythms in resting CV physiology and especially the CV reactivity to stressors; and hypothesize how such circadian rhythms may relate to the established day/night pattern of adverse CV events (7, 9-13). We then examine how chronic disruptions of the internal clock, whether by night-shift work, airline jet lag, or social jet $\operatorname{lag}(20,21)$, are deleterious to CV health. Finally, we highlight new research questions and consider the opportunities for optimizing timing of certain CV medications based on circadian rhythmicity of medication targets (chronotherapy).

\section{Organization of the circadian system}

Circadian timing of physiological function is widespread across the body. In humans and other mammals, the primary circadian pacemaker is the suprachiasmatic nucleus (SCN) of the hypothalamus. In rodents, lesions of the SCN abolish or attenuate daily rhythms in locomotor activity, body temperature, BP, and HR (22-24), showing that these rhythms are endogenous rather than resulting from daily changes in behavior or environment. SCN rhythms are primarily synchronized to the environment by light via the retinohypothalamic tract (25). The SCN is an autonomous oscillator and cycles even in vitro almost indefinitely (26). Moreover, dissociated SCN cells also remain rhythmic in culture, showing that rhythmicity is a cellular property (27). The SCN is critically important in coordinating molecular rhythms in all organs and cells across the body. In all nucleated cells, four families of core clock genes (Clock, Bmal1, Period [Per], and Cryptochrome [Cry]) form a transcriptiontranslation feedback loop that cycles every approximately 24 hours (reviewed in refs. 28-35). CLOCK and BMAL1 act as positive regulators, dimerizing and initiating transcription of the Per and Cry genes. Translated PER and CRY proteins are negative elements in 

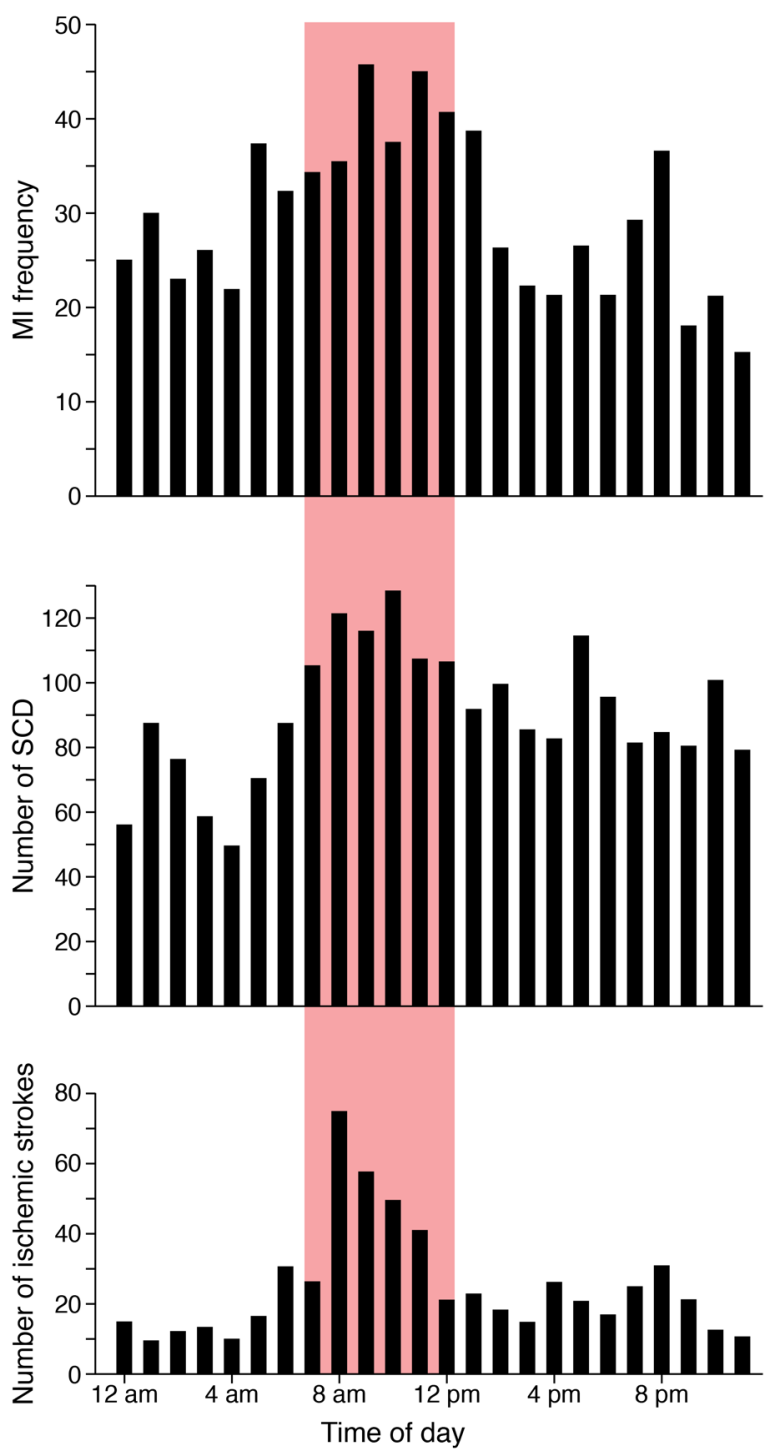

the feedback loop because they inhibit CLOCK::BMAL1 transactivation, and in so doing, shut off their own expression. Other interlocked molecular pathway loops exist including the rhythmic transcription of Rev-erb $\alpha$ and Ror $\alpha$ that in turn control Bmall expression rhythms (36-38). The feedback mechanism encompasses large protein complexes that include chromatin modifiers $(39,40)$, but is still overall poorly understood. In any given mammalian tissue, approximately $5 \%-15 \%$ of gene transcripts are rhythmic, with some differences in the specific cycling genes between tissues, with $43 \%$ of protein-coding genes being rhythmic in at least one tissue (41). These varied tissue clocks are normally entrained by the SCN via neural, endocrine, and physiological pathways (42, 43) including autonomic nervous system projections (44), melato$\operatorname{nin}(45,46)$, glucocorticoids $(47,48)$, body temperature $(49,50)$, and food cues $(51,52)$.

\section{Tissue-specific circadian clocks across the CV system}

In the heart, approximately $13 \%$ of genes and approximately $8 \%$ of proteins are rhythmic (53-56), particularly in growth, metabolism,
Figure 1. The day/night pattern of adverse cardiovascular events. Epidemiological studies reveal that adverse cardiovascular events including myocardial infarction (MI), sudden cardiac death (SCD), and ischemic stroke have increased frequencies in the morning hours compared with other times of the day and night (redrawn from refs. 8, 9, 11).

and molecular signaling pathways $(57,58)$. Combined transcriptomic and metabolic data suggest that each day the cardiomyocyte cycles sequentially through an ATP generation phase to promote contractile function during the active period, then a nutrient storage phase towards the end of the active period, followed by a growth/repair phase in the rest/sleep period $(59,60)$.

Disruptions of the molecular clock in animals cause significant CV disease (61), exemplifying the importance of these rhythms. For instance, Bmal1 knockout in mice causes dilated cardiomyopathy (62) and selective Bmal1 knockout in cardiomyocytes eliminates much of the rhythmic transcriptome (63), reduces stroke volume and ejection fraction, and causes heart failure and early mortality in mice $(63,64)$. BMAL1 is a transcription factor with many genome-binding sites, so some of these results may be due to noncircadian BMAL1 roles, such as abnormal cardiomyocyte metabolism $(63,64)$. In mice, the circadian clock also controls ionic balance in the heart in a healthy range by regulating rhythms in Kruppel-like factor 15 and thereby kChIP2 (a voltage-gated potassium channel), with either deficiency or excess increasing susceptibility to serious ventricular arrhythmias (65). Cardiomyocyte-specific deletion of Bmal1 results in loss of normal circadian expression of the $\mathrm{Na}^{+}$and $\mathrm{K}^{+}$channels resulting in decreased $\mathrm{HR}$ and increased risk of arrhythmias (66), which could contribute to sudden cardiac death (67).

Blood vessels also exhibit circadian rhythmicity. For example, approximately $5 \%$ of all genes in the aorta are rhythmic (68). The vascular endothelium and vascular smooth muscle cells are important in regulating vascular resistance and BP. In animals, genes involved in structural integrity of vascular smooth muscle cells exhibit circadian rhythms (69). Functionally, targeted deletion of Bmal1 in murine vascular smooth muscle cells blunts the circadian amplitudes and advances the circadian phases of systolic and diastolic BP, and abolishes the circadian variation of pulse pressure (70).

The kidney is essential to long-term regulation of $\mathrm{BP}$ and approximately $13 \%$ of protein-coding genes in the kidney are rhythmic (41). PER1 plays an important role in BP maintenance by regulating aldosterone levels and affecting sodium retention (71-73). Bmal1-knockout mice exhibit lower BP with no circadian variation (74) perhaps in part because of the arrhythmicity in behaviors, including locomotor activity, in mice $(75,76)$. Patients with chronic kidney disease commonly lack the normal greater than $10 \%$ dip in nighttime BP compared with daytime BP. This so-called "non-dipping BP profile" $(77,78)$, which is associated with increased risk of adverse $\mathrm{CV}$ events and death in people with hypertension $(79,80)$. The precise role of the circadian system in nondipping hypertension is yet to be elucidated.

\section{Circadian rhythms in CV function}

Dissociating circadian, behavioral, and environmental effects. To estimate the contribution of the endogenous circadian system to 


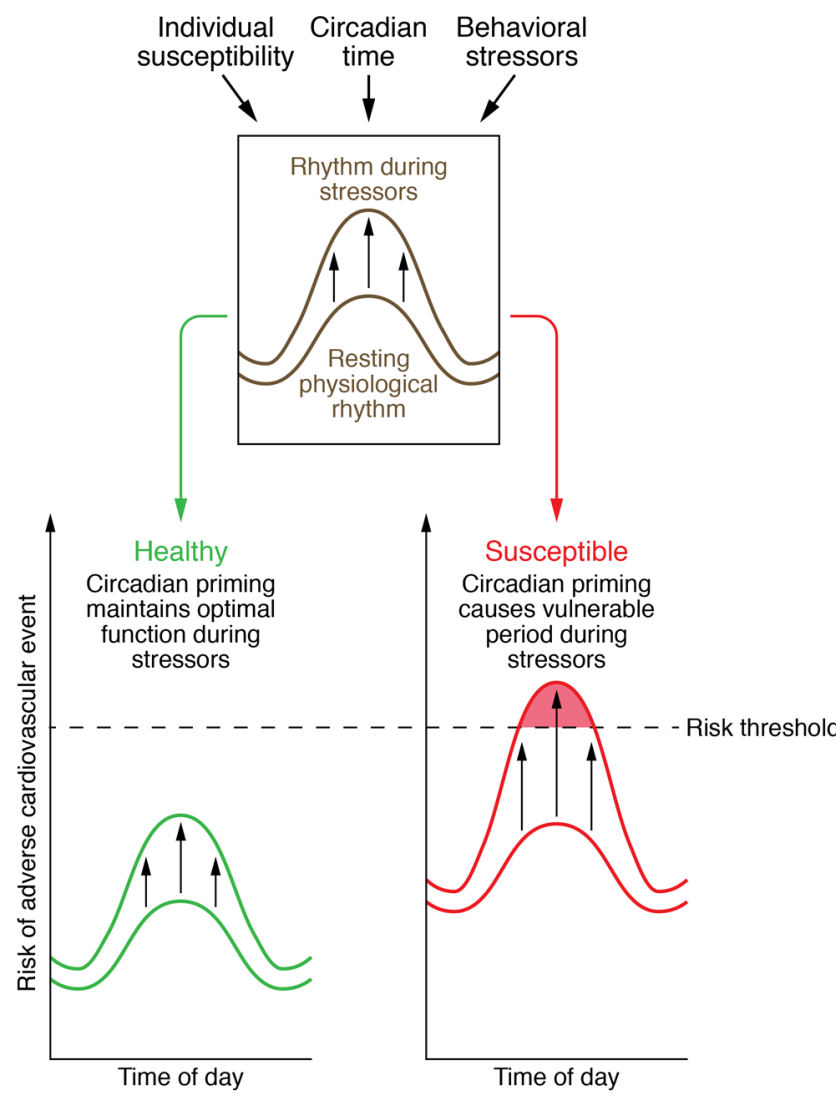

CV physiology, two classic protocols have been used that experimentally dissociate circadian clock effects from rhythmic behavioral and environmental effects (81). The first is the constant routine (82), in which environmental, behavioral, and postural changes are minimized for at least 24 hours. In our hands, participants maintained wakefulness while lying in a semirecumbent posture and consuming identical isocaloric snacks every 2 hours while in dim light, constant temperature, and with an absence of external time cues (83-86). In the constant routine, we observed clear circadian rhythms in many variables (83-86), but there are also potential changes caused by accumulating sleep loss $(87,88)$. These two effects may be mathematically separated based on assumptions of an underlying 24-hour circadian sinusoidal rhythmicity that is superimposed on a monotonic effect of accumulating sleep loss (84). The second protocol is the forced desynchrony; it is implemented across multiple days and avoids the problem of accumulating sleep loss (Figure 3). Participants live on non-24hour cycles of sleep and wakefulness with periods sufficiently far from 24 hours that their internal clocks cannot synchronize to the imposed artificial day length. We have used imposed behavioral cycles of 5 hours 20 minutes (89), 20 hours $(90,91)$, and 28 hours (92). The behaviors (sleep/wake, fasting/feeding, and inactivity/ activity) therefore become desynchronized from the normal 24-hour schedule and thereby desynchronized from the endogenous circadian clock (Figure 3). By standardizing and balancing all behaviors evenly across the whole circadian cycle, a forced desynchrony protocol permits statistical determination of (a) an average circadian rhythm while controlling for all behavior effects (by aligning data according to the period of the circadian pacemaker
Figure 2. Conceptual model showing interactions between behavioral stressors, circadian timing, and individual susceptibility to cardiovascular risk. There is a constant interaction between behavioral stressors (e.g. exercise), circadian phases, and underlying individual risk. This results in resting physiological rhythms (e.g., blood pressure) and reactivity of those rhythms in the presence of stressors. In a healthy individual, the resting physiological rhythms are within normal limits and the reactivity to a stressor (upward-pointing arrows) is modest, such that the theoretical risk threshold for an adverse cardiovascular event is not crossed. However, in an individual susceptible to cardiovascular risk due to existing anatomical or physiological maladaptation, resting physiological rhythms may be elevated. Furthermore, in the presence of a stressor, the reactivity of physiological rhythms may be exaggerated and can cross the theoretical risk threshold for an adverse cardiovascular event such as stroke.

at approximately 24 hours); (b) an average effect of a standardized behavior while controlling for any underlying circadian effects (by aligning data according to the period of the imposed artificial day length); and (c) any interaction between these effects, effectively determining if the responses to a behavior are different at different times of the body clock cycle $(90,91)$. In humans, food timing in these protocols does not appear to affect central circadian rhythms (18), but the effects on peripheral oscillators, for instance in adipose tissue or the liver, are unknown. In animals, timed feeding is a strong resetting cue for peripheral clocks $(51,52)$. To date, there have been far fewer investigations in humans, and any resetting effects of meals appear much smaller (93).

Circadian rhythm in hemodynamic regulation. Systemic $\mathrm{BP}$ is a ubiquitous measure in the clinic but is far from static across the day and night, and this day/night pattern of BP has clear clinical relevance. BP is usually lower in sleep than in wakefulness and has a characteristic surge after awakening, drawing parallels with the daily pattern of adverse CV events (refs. 94-97 and Figure 1). Indeed, in elderly patients with hypertension, a 10-mmHg systolic BP surge within 2 hours after waking is associated with a $22 \%$ greater risk of stroke, independent of the 24-hour average BP (98). The morning BP surge is not the only risk, as high nighttime BP (lack of nocturnal BP dipping) is a sensitive indicator for CV mortality, with $21 \%$ greater risk for each $10-\mathrm{mmHg}$ increase in nighttime systolic BP and 9\% greater risk for each 5-mmHg increase in nighttime diastolic BP (99).

Very few studies have measured the relative contributions of the daily pattern of behaviors and of the circadian clock to this day/ night pattern in BP in humans. Two early studies that used 24-hour or 26-hour modified constant routine protocols reported no circadian rhythmicity of BP $(100,101)$. However, those studies had limitations, including exposure to light (which could affect circadian phase, ref. 102; suppress melatonin, ref. 103; and reduce potential melatonin-mediated dips in BP, ref. 104), lack of strict control of physical activity (which could introduce variable effects on BP), and lack of a circadian phase marker. In contrast, using three stringently controlled constant routine and forced desynchrony protocols that overcame these limitations, robust endogenous circadian rhythms in BP were discovered $(83,90)$. The endogenous circadian resting $\mathrm{BP}$ rhythm has an evening peak and a morning trough. Theoretically, the morning circadian trough could be protective by partially counteracting the BP surges related to arousal from sleep, sudden change in posture on awakening, and transient increase in 
A

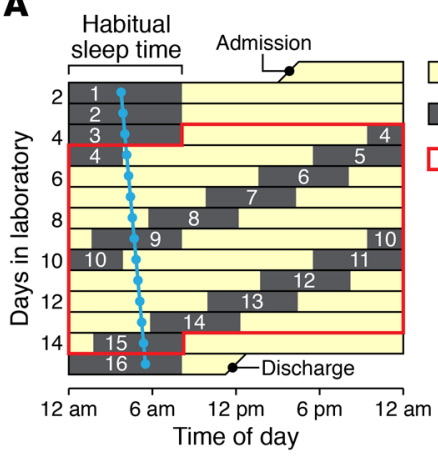

B

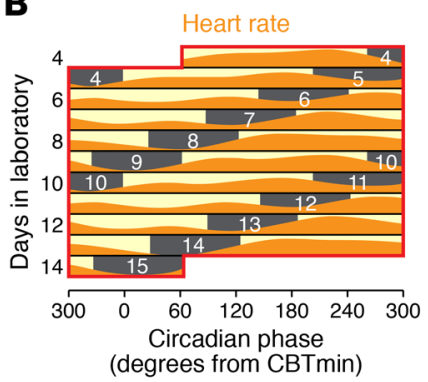

C

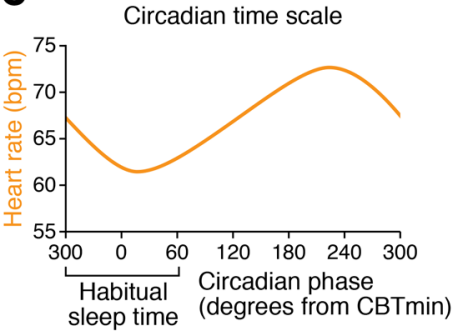

Figure 3. Example of a forced desynchrony protocol to separate the effects of the endogenous circadian system from the effects of daily behaviors. (A) Over 15 days, lights are dim during wake periods and off during sleep periods to allow the circadian clock to tick at its own rate (usually slightly longer than 24 hours - shown here by the slowly drifting core body temperature minimum [CBTmin]). During the forced desynchrony portion (boxed in red), participants experience recurrent identical sleep/ wake cycles evenly spread across the circadian cycle (here, each sleep episode is 6.67 hours and wake episode is 13.33 hours). Scheduled wake episodes are shown in yellow and sleep episodes are shaded gray. (B) Data are collected across the protocol. Model heart rate data are shown for one trial and plotted as a function of circadian time (left) or of time into each imposed 20-hour sleep/wake cycle (right). Note the relatively high heart rate during the wake episodes versus sleep episodes. (C) Raw data are averaged across different time scales to reveal endogenously rhythmic components and behaviorally elicited components. Here, despite identically scheduled behaviors in each wake episode, there is an overall approximately 24-hour rhythm, with low heart rate at the time of habitual sleep (left). As expected, heart rate is lower during (actual) sleep episodes than during waking episodes (right).

dian rhythm of systolic BP (90). On the other hand, the efferent cardiac vagal tone (estimated from HR variability) had a circadian peak at approximately $8 \mathrm{am}$, coincident with the circadian basal BP trough (90), suggesting that circadian rhythms of BP and parasympathetic nervous system activity may be mechanistically related in resting healthy individuals.

In health, heartbeat dynamics exhibit complex fluctuations characterized by robust self-similar temporal structures, i.e., fractal regulation (108). A study using a forced desynchrony discovered that these temporal correlations have a circadian rhythm (independent from scheduled behaviors, activity, and mean HR) with signs of breaking down towards patterns seen in congestive heart failure during the vulnerable morning period (108). This raises the possibility that circadian-mediated influences on cardiac dynamics are involved in the day/night pattern of adverse cardiac events (107).

Circadian rhythm in hemostatic regulation. The ability to clot blood can be life-saving after an injury, but thrombi within vessels can contribute to myocardial infarction, ischemic stroke, and sudden cardiac death. Fibrinolytic activity helps break down thrombi and maintain vessel patency. The dynamic balance of platelet activation and fibrinolysis across the day and night may be involved in the morning increased risk for occlusive thrombi. For instance, highest platelet aggregability and highest concentration of plasminogen activator inhibitor-1 (PAI-1) occur in the morning $(109,110)$. A forced desynchrony protocol revealed that PAI- 1 is rhythmic and peaks in the biological morning, suggesting a role for the circadian clock in reducing fibrinolytic activity in the morning, especially since this circadian rhythm was approximately 8 times larger than changes in PAI-1 induced by behaviors such as mild exercise (111). Indeed, several basic studies have demonstrated that PAI-1 gene expression is under molecular clock control (112-114). There are also effects of the circadian system on platelet activation, with a morning peak in platelet surface-activated GPIIb-IIIa, the final part of the platelet aggregation pathway (115). Thus, the circadian system appears to prepare healthy humans for increased ability to clot blood and reduce the breakdown of clots in the morning, with obvious potential clinical relevance in susceptible individuals. We speculate that 
evolution has led to increased ability to clot blood in anticipation of increased laceration risk after awakening and becoming active. Yet, in people with $\mathrm{CV}$ vulnerabilities, increased clotting in the morning could increase the risk for adverse CV events (111). Indeed, aspirin reduces the morning peak in myocardial infarction (116), suggesting that the prothrombotic milieu in the morning is partly responsible for increased CV risk during that period.

Day/night pattern of vascular endothelial function. The vascular endothelium exerts remarkable control over the CV system, performing anti-atherosclerotic functions and regulating vascular patency via secretion of nitric oxide (117-119). A dysfunctional vascular endothelium is prognostic of adverse CV events (120). In animal studies, deletion or mutation of the core clock gene Per2 impairs acetylcholine-induced endothelial relaxation (121), impairs endothelial proliferation, and promotes endothelial aging (122). In humans, vascular endothelial function is relatively impaired in the morning $(6,123-126)$, and therefore potentially implicated in the morning increase in adverse CV events (127). Interestingly, in people with severe heart failure, endothelial function is impaired at all times, with loss of daily variation in endothelial function (128). Jones et al. (129) found decreased endothelial function across naps in the afternoon and at night, suggesting that either sleep or the inactivity that accompanies sleep accounts for the documented morning impairment in endothelial function $(6,123-126)$, although endogenous circadian system effects have not yet been appropriately investigated in healthy people or those with CV disease.

Circadian rhythms in $\mathrm{CV}$ reactivity to behaviors. While there is clear evidence of basal circadian rhythms in the CV system, reactions to physiological stressors are more likely to reveal vulnerabilities. Thus, interactions between the circadian system and the acute $\mathrm{CV}$ reactions to stressors may better exemplify the circadian system's importance (Figure 2). Indeed, rather than a simple summation of circadian and behavioral effects, the same stressor may produce different CV responses at different circadian times (90, 91). Such nonlinear interactions presumably optimize function in healthy individuals but could theoretically set up the possibility of adverse $\mathrm{CV}$ events in susceptible individuals (Figures 1 and 2). For instance, ambulatory BP responses to acute increases in activity are higher in the morning (14), which may be optimal in physically active individuals but could trigger adverse CV events in habitually inactive individuals (130).

Since changes in posture and activity both usually occur during the vulnerable morning period following sleep, we determined whether $\mathrm{CV}$ responses to postural changes and exercise differ across the circadian cycle $(90,91)$. A 20-hour forced desynchrony in healthy individuals with tilt table tests $\left(60^{\circ}\right.$ head-up for $15 \mathrm{~min}$ utes) across all phases of the circadian cycle revealed presyncope in $15 \%$ of all tests, with almost all cases $(81 \%)$ occurring during the half of the circadian cycle corresponding to the biological night (2230-1030 hours) (91). Prior to that study, tilt table tests had rarely, if ever, been performed during the night, so this nocturnal period of increased vulnerability went unnoticed. This vulnerable period could have implications for individuals who become active during the night, such as shift workers, parents feeding their infants, and elderly people with increased nocturia and insomnia. In the identical 20-hour forced desynchrony it was found that the circadian system significantly modulated the vagal, sympathetic, and BP reactiv- ity to mild cycle exercise (90). Notably, exercise-induced increases in epinephrine were twice as large in the morning (0830 hours) than in the middle of the night (0430 hours) (90). There were also significant circadian rhythms in the reactivity of parasympathetic markers, with the greatest cardiac vagal withdrawal during exercise across the morning period. These autonomic changes in the morning also corresponded to the circadian phase with the smallest systolic BP reactivity to cycle exercise (90). Rather than a simple 24-hour rhythm, the reactivities to exercise of both epinephrine and norepinephrine exhibited 12-hour rhythms with large peaks at circadian phases corresponding to 0700-1030 and 2000-2200 hours. Such 12-hour rhythms in reactivity to exercise could be related to 12-hour rhythms demonstrated in animal studies in, for instance, cardiac $\beta$-adrenergic receptor density and function (131), and gene transcription in liver, heart, kidney, and adrenals (132).

In summary, healthy people exhibit greatest sympathetic activation and greatest parasympathetic withdrawal during stresses in the biological morning, which corresponds to the time of the greatest risk of adverse events based on epidemiological data. Similar circadian CV studies are warranted in people with CV disease.

\section{Night-shift work, circadian disruption, and social jet lag}

Nowadays, the availability of artificial light at the flick of a switch provides the opportunity to adjust endogenous clock timing to a newly imposed light/dark cycle (e.g., night-shift work or jet lag) (25). However, repeated shifting of the endogenous circadian clock is injurious to health. Shift work is an independent risk factor for many chronic conditions, including heart disease (133-136), perhaps due to recurring disruptions of the circadian clock itself (e.g., light exposure at unusual circadian phases), or perhaps due to misalignment between the circadian clock and the behaviors (e.g., sleeping and fasting during the day, and being active and eating across the night). In humans, transient misalignment of behaviors and the circadian clock increases inflammation and impairs glucose regulation (137-140), with potential relevance to CV disease (141). A recent laboratory study of shift workers revealed that circadian misalignment increased 24-hour BP and CV inflammatory markers including IL-6, C-reactive protein, and TNF- $\alpha$ (142). Though similar measures after chronic circadian disruption are not available in humans, results in animal models suggest serious long-term consequences. Weekly 12-hour phase shifts of the light/ dark cycle reduced longevity in cardiomyopathic hamsters by $11 \%$ (143). Cardiomyopathy was observed in hamsters whose internal circadian clock period did not match the external 24-hour light cycle (i.e., 20-hour period due to the tau mutation) (61,144). Circadian disruption also slows recovery from CV events. For instance, recovery from experimentally induced myocardial ischemia is impaired in mice by rapidly shifting light/dark cycles, suggesting that sleep and circadian rhythm disruption that occurs in intensive care units may compromise recovery (145). Genetic manipulation of clock components impacts the ischemic tolerance of the heart, with some mutations worsening (146) and some improving the response (147). Thus, both external (light/dark cycle shifts) and internal (molecular clock disruption) impact CV health in mice.

Appropriately shifting circadian phase to match night-shift work is desirable but difficult and never immediate. Use of 
bright light during night shifts and reducing light exposure following the end of night shifts (148-150) helps shift the circadian pacemaker and does improve performance, mood, and reduces sympathetic activity in night-shift workers who sleep during the daytime (151). However, in practice, daily light exposure, family and social demands, conflicting time cues on days off, and rapidly rotating shifts make appropriate circadian synchronization to the work schedule quite challenging (152). This means that work, meals, and sleep consistently occur at suboptimal circadian times. Clock-environment misalignment or clock-behavior misalignment are not limited to night-shift workers. A smaller but similar clock disruption is termed social jet lag, defined as the difference in the time of sleep between work/school days and free days (153). Approximately $70 \%$ of the population have noticeable social jet lag (i.e., their biological and social clocks differ by more than 1 hour) (154), and the greater the social jet lag, the greater the risk for obesity and diabetes (154-156). The effects of social jet lag on the CV system have not been well studied, though it is reported that greater social jet lag is associated with increased $\mathrm{HR}$ and higher cortisol levels (155). Moreover, a mere 1-hour advance in behaviors relative to circadian time, as occurs with a shift to daylight savings time in the spring, is associated with a transient increase in the incidence of myocardial infarctions (157). Applying circadian principles, such as appropriately timed light, darkness, and sleep, may help minimize circadian misalignment during night-shift work, but these circadian phase shifts are generally small and take numerous days to achieve such that some compromise seems inevitable (158). Thus, research on rapidly shifting the phase of the body clock to reduce misalignment is warranted. Indeed, the recent discovery that blockade of vasopressin receptors in the SCN accelerated recovery from jet lag in mice is promising in this regard (159).

\section{Future research}

The genes, receptors, cells, organs, and baseline physiology of the CV system all exhibit marked internal circadian rhythmicity. However, it is the interplay between these baseline circadian rhythms and the reactivity to daily changes in behavior or the environment that likely holds the key to answering whether and how circadian rhythms benefit the organism. It is also of interest to determine whether 24-hour circadian changes in CV variables follow the simple negative-feedback regulation principles that are well described for the CV system. For instance, acutely increased sympathetic activation invariably leads to increased HR; therefore, should we expect that a circadian peak in circulating catecholamines will cause a circadian peak in HR, or will a different dynamic relationship occur at these longer time scales? In animal models, it has been demonstrated that circadian rhythms in physiology are altered in disease states. Yet, modelling circadian disruption in humans is difficult, and most experiments have been performed on healthy participants. Therefore, a critical gap exists in the identification of the potential mechanisms by which the rhythms in CV physiology in healthy participants translate to people with existing $\mathrm{CV}$ disease. In particular, do normal physiological responses expose vulnerabilities at specific circadian phases in people made susceptible to adverse CV events because of underlying disease (Figure 2)? Moreover, it will be worthwhile to determine if CV circadian rhythms are affected by chronic $\mathrm{CV}$ disease. Another area for future exploration is to determine if the well-described racial disparities in CV risk (160) can be explained by potential racial differences in endogenous circadian rhythms (161).

Circadian clocks are very precise under constant conditions but can be reset using external stimuli. A promising area of clinical research is the use of small-molecule modifiers that target the circadian clock to reduce pathology, such as KL001 for diabetes (162) and nobiletin for metabolic syndrome (163). In animal models, REV-ERB $\alpha$ has been shown to phase shift molecular clocks (164). A synthetic REV-ERB agonist taken by mice with diet-induced obesity reversed this obesity by reducing fat mass and improving dyslipidemia and hyperglycemia (165), while REV-ERB $\alpha$ upregulation increased muscle mitochondrial content and oxidative capacity (166). Conversely, in a clinical trial of timing of aortic valve replacement, perioperative myocardial injury was significantly lower in those undergoing surgery in the afternoon compared with the morning when myocardial REB-ERB $\alpha$ was highest (167).

\section{Clinical directions: chronotherapy}

The existence of clear epidemiological patterns of adverse CV events and the increase in CV risk in the face of abnormal diurnal patterns in physiology, such as the nondipping hypertension profile $(77,168)$, underline the importance of considering new time-based approaches to treat CV disease. A host of CV medications prescribed to help patients recover from and/or prevent heart disease include antithrombotics, $\beta$-adrenoreceptor antagonists, calcium channel blockers, nitrates, angiotensin-converting enzyme (ACE) inhibitors, angiotensin receptor blockers, and cholesterol-lowering agents. Moreover, depending on specific CV diseases, their severity, and any comorbidities, most patients receive more than one medication (169). The goal of medication dosing is to optimize effectiveness without causing serious adverse side effects, both of which may depend on circadian time $(170,171)$. Furthermore, pharmacokinetics are driven by the absorption, distribution, metabolism, and excretion of a medication, each of which may be modulated by circadian rhythms (172). Indeed, gene expression is rhythmic for the targets of the majority of top-selling and essential medications listed by the World Health Organization (41), including angiotensin II receptor blockers, $\beta$-adrenoreceptor antagonists, and aldosterone receptor blockers (41). Therefore, the field of chronotherapy - which considers the timing of medication doses relative to internal circadian phase, or more commonly the time of day - holds promise to improve care.

There are some classic examples where the time of CV medication administration resulted in differential effects on CV function (173). Use of $\beta$-adrenoreceptor blockers reduces adverse $\mathrm{CV}$ events on an overall-population basis and abolishes the day/ night pattern of such events $(9,174)$. It has been recommended to take extended-release $\beta$-adrenoreceptor blockers in the evening, as this is sufficient to keep levels high during the morning vulnerable period (175). The morning surge of BP can be reduced in hypertensive patients by bedtime administration of a longacting lipophilic ACE inhibitor (176). Likewise, low-dose aspirin can reduce the morning peak in myocardial infarction by approximately $60 \%$ (116), with evening aspirin theoretically being best to reduce potential for thrombus formation across the morning vulnerable period, although evidence from placebo-controlled 
trials with long-term follow up of CV outcomes is lacking (177). Interestingly, in people with mild untreated hypertension, 100 mg aspirin at bedtime reduced 24-hour systolic BP by an average of $7 \mathrm{mmHg}$ and diastolic BP by $5 \mathrm{mmHg}$, whereas a similar dose of aspirin upon awakening actually increased 24-hour systolic BP by an average of $3 \mathrm{mmHg}$ and diastolic BP by $2 \mathrm{mmHg}$ (178). Of note, mouse expression of cyclooxygenase-1 (COX-1), a target for aspirin, exhibits circadian rhythms in several tissues including the heart (41). Another study of the ACE inhibitor captopril showed that it reduced BP in mice at all times of day, but improved cardiac function only when administered at sleep time, potentially owing to captopril administration being in phase with increased ACE in the heart, and a consequent time-gated cardiac remodeling (179).

The MAPEC study of over 2,000 individuals with resistant hypertension is one of the few chronotherapeutic trials with longterm CV outcomes in humans (180). Patients in the MAPEC study were randomized to either change the timing of the dose of at least one antihypertensive medication to bedtime, or to maintain their usual practice of taking all hypertensive medications in the morning (180). Evening dosing reduced incidence of all CV events and CV mortality during 5.6 years of follow-up. These results were replicated in people with type II diabetes (181). While highly promising, the mechanisms are not yet understood, and doubleblind placebo-controlled trials are now warran8ted with specific hypertensive medications rather than any hypertensive medications. In any chronotherapeutic trial, it should be noted that the magnitude of side effects can also depend on the time of dosing. For instance, a minor concern for evening chronotherapy using $\beta$-adrenoreceptor blockers is that these medications can suppress normal melatonin release, which may affect sleep (182).

As the science of chronotherapeutics develops, we hope to move from simply prescribing medications relative to clock time (e.g., morning or evening with respect to our watches), or relative to daily behavioral patterns (e.g., before bedtime, or with a meal) to prescribing medications relative to endogenous biological time (i.e., circadian phases) and with possible consideration of any interaction between circadian phase and behavioral cycle on the effects of medications. These considerations can be important because internal circadian phase relative to external time or behavioral cycle varies between people, across ages, and depends on habitual light exposure (e.g., night-shift work). Moreover, if an evening dose is found effective, it will be important to determine if this is due to the circadian clock, for example via its regulation of a specific medication target that has circadian rhythmicity, or due to behaviors, such as meal history. Physicians may also wish to consider the possibility that comorbid conditions may affect the effectiveness of chronotherapy. For example, the vulnerable period for an adverse $\mathrm{CV}$ event in the general population is in the morning, coinciding with a morning surge in BP (183), but in patients with long-term ( $\geq 5$ years) type I or II diabetes, the morning peak in myocardial infarction is significantly blunted (184). Similarly, the vulnerable period for sudden cardiac death is relatively advanced to the middle of the night rather than the morning in people with obstructive sleep apnea (185). Finally, we acknowledge that dosing according to internal circadian phase is presently somewhat impractical because estimation of internal phase (e.g., from serial salivary melatonin assays) is slow and expensive. Thus, development of an inexpensive, accurate, and instantaneous biomarker of internal circadian phase is a major challenge for the field.

\section{Acknowledgments}

The authors acknowledge funding from the National Space Biomedical Research Institute through NCC 9-58; NIH grants R01HL125893, R01HL140577, R01HL142064, R21HL140377, and F32HL131308; the Oregon Institute of Occupational Health Sciences; the Medical Research Foundation of Oregon; and the American Sleep Medicine Foundation.

Address correspondence to: Steven A. Shea, Oregon Health and Science University, Mail Code L606, 3181 SW Sam Jackson Park Road, Portland, Oregon 97239, USA. Phone: 503.494.2517; Email: sheast@ohsu.edu.
1. Weber MA, Drayer JI, Nakamura DK, Wyle FA. The circadian blood pressure pattern in ambulatory normal subjects. Am J Cardiol. 1984;54(1):115-119.

2. Millar-Craig M, Bishop C, Raftery E. Circadian variation of blood-pressure. Lancet. 1978;1(8068):795-797.

3. Degaute JP, van de Borne P, Linkowski P, Van Cauter E. Quantitative analysis of the 24-hour blood pressure and heart rate patterns in young men. Hypertension. 1991;18(2):199-210.

4. Turton MB, Deegan T. Circadian variations of plasma catecholamine, cortisol and immunoreactive insulin concentrations in supine subjects. Clin Chim Acta. 1974;55(3):389-397.

5. Angleton P, Chandler WL, Schmer G. Diurnal variation of tissue-type plasminogen activator and its rapid inhibitor (PAI-1). Circulation. 1989;79(1):101-106.

6. Otto ME, et al. Early morning attenuation of endothelial function in healthy humans. Circulation. 2004;109(21):2507-2510.

7. Elliott WJ. Circadian variation in the tim- ing of stroke onset: a meta-analysis. Stroke. 1998;29(5):992-996.

8. Manfredini R, et al. Circadian variation in stroke onset: identical temporal pattern in ischemic and hemorrhagic events. Chronobiol Int. 2005;22(3):417-453.

9. Muller JE, et al. Circadian variation in the frequency of onset of acute myocardial infarction. N Engl J Med. 1985;313(21):1315-1322.

10. Goldberg RJ, et al. Time of onset of symptoms of acute myocardial infarction. Am JCardiol. 1990;66(2):140-144.

11. Muller JE, Tofler GH, Willich SN, Stone PH. Circadian variation of cardiovascular disease and sympathetic activity. J Cardiovasc Pharmacol. 1987;10(suppl 2):S104-S109; discussion S110.

12. Twidale N, Taylor S, Heddle WF, Ayres BF, Tonkin AM. Morning increase in the time of onset of sustained ventricular tachycardia. Am J Cardiol. 1989;64(18):1204-1206.

13. Willich SN, Goldberg RJ, Maclure M, Perriello $\mathrm{L}$, Muller JE. Increased onset of sudden cardiac death in the first three hours after awakening. Am
J Cardiol. 1992;70(1):65-68

14. Jones H, Atkinson G, Leary A, George K, Murphy M, Waterhouse J. Reactivity of ambulatory blood pressure to physical activity varies with time of day. Hypertension. 2006;47(4):778-784.

15. Leor J, Poole WK, Kloner RA. Sudden cardiac death triggered by an earthquake. $N$ Engl J Med. 1996;334(7):413-419.

16. Muller JE, Tofler GH, Verrier RL. Sympathetic activity as the cause of the morning increase in cardiac events. A likely culprit, but the evidence remains circumstantial. Circulation. 1995;91(10):2508-2509.

17. Feng DL, Tofler GH. Diurnal physiologic processes and circadian variation of acute myocardial infarction. JCardiovasc Risk. 1995;2(6):494-498.

18. Czeisler CA, et al. Stability, precision, and near24-hour period of the human circadian pacemaker. Science. 1999;284(5423):2177-2181.

19. Kirschbaum C, Wüst S, Hellhammer D. Consistent sex differences in cortisol responses to psychological stress. Psychosom Med. 1992;54(6):648-657. 
20. Rutters F, et al. Is social jetlag associated with an adverse endocrine, behavioral, and cardiovascular risk profile? J Biol Rhythms. 2014;29(5):377-383.

21. Kawachi I, et al. Prospective study of shift work and risk of coronary heart disease in women. Circulation. 1995;92(11):3178-3182.

22. Ralph MR, Foster RG, Davis FC, Menaker M. Transplanted suprachiasmatic nucleus determines circadian period. Science. 1990;247(4945):975-978.

23. Stephan FK, Zucker I. Circadian rhythms in drinking behavior and locomotor activity of rats are eliminated by hypothalamic lesions. Proc Natl Acad SciU S A. 1972;69(6):1583-1586.

24. Witte K, et al. Effects of SCN lesions on circadian blood pressure rhythm in normotensive and transgenic hypertensive rats. Chronobiol Int. 1998;15(2):135-145.

25. Gooley JJ, Lu J, Chou TC, Scammell TE, Saper CB. Melanopsin in cells of origin of the retinohypothalamic tract. Nat Neurosci. 2001;4(12):1165.

26. Yamazaki S, Takahashi JS. Real-time luminescence reporting of circadian gene expression in mammals. Meth Enzymol. 2005;393:288-301.

27. Welsh DK, Takahashi JS, Kay SA. Suprachiasmatic nucleus: cell autonomy and network properties. Annu Rev Physiol. 2010;72:551-577.

28. Takahashi JS. Molecular components of the circadian clock in mammals. Diabetes Obes Metab. 2015;17(Suppl 1):6-11.

29. van der Horst GT, et al. Mammalian Cry1 and Cry2 are essential for maintenance of circadian rhythms. Nature. 1999;398(6728):627-630.

30. Tei H, et al. Circadian oscillation of a mammalian homologue of the Drosophila period gene. Nature. 1997;389(6650):512-516.

31. Sun ZS, Albrecht U, Zhuchenko O, Bailey J, Eichele G, Lee CC. RIGUI, a putative mammalian ortholog of the Drosophila period gene. Cell. 1997;90(6):1003-1011.

32. Kume K, et al. mCRY1 and mCRY2 are essential components of the negative limb of the circadian clock feedback loop. Cell. 1999;98(2):193-205.

33. Konopka RJ, Benzer S. Clock mutants of Drosophila melanogaster. Proc Natl Acad Sci U S A. 1971;68(9):2112-2116.

34. Gekakis N, et al. Role of the CLOCK protein in the mammalian circadian mechanism. Science. 1998;280(5369):1564-1569.

35. Vitaterna $\mathrm{MH}$, et al. Mutagenesis and mapping of a mouse gene, Clock, essential for circadian behavior. Science. 1994;264(5159):719-725.

36. Akashi M, Takumi T. The orphan nuclear receptor RORalpha regulates circadian transcription of the mammalian core-clock Bmal1. Nat Struct Mol Biol. 2005;12(5):441-448.

37. Sato TK, et al. A functional genomics strategy reveals Rora as a component of the mammalian circadian clock. Neuron. 2004;43(4):527-537.

38. Preitner $\mathrm{N}$, et al. The orphan nuclear receptor REV-ERBalpha controls circadian transcription within the positive limb of the mammalian circadian oscillator. Cell. 2002;110(2):251-260.

39. Duong HA, Weitz CJ. Temporal orchestration of repressive chromatin modifiers by circadian clock Period complexes. Nat Struct Mol Biol. 2014;21(2):126-132.

40. Aryal RP, et al. Macromolecular assemblies of the mammalian circadian clock. Mol Cell. 2017;67(5):770-782.e6

41. Zhang R, Lahens NF, Ballance HI, Hughes $\mathrm{ME}$, Hogenesch JB. A circadian gene expression atlas in mammals: implications for biology and medicine. Proc Natl Acad Sci U S A 2014;111(45):16219-16224.

42. Dibner C, Schibler U, Albrecht U. The mammalian circadian timing system: organization and coordination of central and peripheral clocks. Annu Rev Physiol. 2010;72:517-549.

43. Mohawk JA, Green CB, Takahashi JS. Central and peripheral circadian clocks in mammals. Annu Rev Neurosci. 2012;35:445-462.

44. Ueyama T, et al. Suprachiasmatic nucleus: a central autonomic clock. Nat Neurosci. 1999;2(12):1051-1053.

45. von Gall C, Weaver DR, Moek J, Jilg A, Stehle JH, Korf HW. Melatonin plays a crucial role in the regulation of rhythmic clock gene expression in the mouse pars tuberalis. Ann N Y Acad Sci. 2005;1040:508-511.

46. Valenzuela FJ, et al. Clock gene expression in adult primate suprachiasmatic nuclei and adrenal: is the adrenal a peripheral clock responsive to melatonin? Endocrinology. 2008;149(4):1454-1461.

47. Balsalobre A, et al. Resetting of circadian time in peripheral tissues by glucocorticoid signaling. Science. 2000;289(5488):2344-2347.

48. Cuesta M, Cermakian N, Boivin DB. Glucocorticoids entrain molecular clock components in human peripheral cells. FASEB J. 2015;29(4):1360-1370.

49. Brown SA, Zumbrunn G, Fleury-Olela F, Preitner $\mathrm{N}$, Schibler U. Rhythms of mammalian body temperature can sustain peripheral circadian clocks. Curr Biol. 2002;12(18):1574-1583.

50. Buhr ED, Yoo SH, Takahashi JS. Temperature as a universal resetting cue for mammalian circadian oscillators. Science. 2010;330(6002):379-385.

51. Damiola F, Le Minh N, Preitner N, Kornmann B, Fleury-Olela F, Schibler U. Restricted feeding uncouples circadian oscillators in peripheral tissues from the central pacemaker in the suprachiasmatic nucleus. Genes Dev. 2000;14(23):2950-2961.

52. Stokkan KA, Yamazaki S, Tei H, Sakaki Y, Menaker M. Entrainment of the circadian clock in the liver by feeding. Science. 2001;291(5503):490-493.

53. Storch KF, et al. Extensive and divergent circadian gene expression in liver and heart. Nature. 2002;417(6884):78-83.

54. Martino T, et al. Day/night rhythms in gene expression of the normal murine heart. J Mol Med. 2004;82(4):256-264.

55. Tsimakouridze EV, et al. Chronomics of pressure overload-induced cardiac hypertrophy in mice reveals altered day/night gene expression and biomarkers of heart disease. Chronobiol Int. 2012;29(7):810-821.

56. Podobed P, et al. The day/night proteome in the murine heart. Am J Physiol Regul Integr Comp Physiol. 2014;307(2):R121-R137.

57. Martino TA, et al. Disturbed diurnal rhythm alters gene expression and exacerbates cardiovascular disease with rescue by resynchronization. Hypertension. 2007;49(5):1104-1113.

58. Martino T, et al. Day/night rhythms in gene expression of the normal murine heart. JMo Med. 2004;82(4):256-264.

59. Durgan DJ, et al. O-GlcNAcylation, novel posttranslational modification linking myocardial metabolism and cardiomyocyte circadian clock. J Biol Chem. 2011;286(52):44606-44619.

60. Zhang L, et al. KLF15 establishes the landscape of diurnal expression in the heart. Cell Rep. 2015;13(11):2368-2375.

61. Martino TA, et al. Circadian rhythm disorganization produces profound cardiovascular and renal disease in hamsters. Am J Physiol Regul Integr Comp Physiol. 2008;294(5):R1675-R1683.

62. Lefta M, Campbell KS, Feng HZ, Jin JP, Esser KA. Development of dilated cardiomyopathy in Bmal1-deficient mice. Am J Physiol Heart Circ Physiol. 2012;303(4):H475-H485.

63. Young ME, et al. Cardiomyocyte-specific BMAL1 plays critical roles in metabolism, signaling, and maintenance of contractile function of the heart. JBiol Rhythms. 2014;29(4):257-276.

64. Bray MS, et al. Disruption of the circadian clock within the cardiomyocyte influences myocardial contractile function, metabolism, and gene expression. Am J Physiol Heart Circ Physiol. 2008;294(2):H1036-H1047.

65. Jeyaraj D, et al. Circadian rhythms govern cardiac repolarization and arrhythmogenesis. Nature. 2012;483(7387):96-99.

66. Schroder EA, et al. The cardiomyocyte molecular clock, regulation of Scn5a, and arrhythmia susceptibility. Am J Physiol Cell Physiol. 2013;304(10):C954-C965.

67. Schroder EA, et al. The cardiomyocyte molecular clock regulates the circadian expression of Kcnh2 and contributes to ventricular repolarization. Heart Rhythm. 2015;12(6):1306-1314.

68. Rudic RD, et al. Bioinformatic analysis of circadian gene oscillation in mouse aorta. Circulation. 2005;112(17):2716-2724.

69. Chalmers JA, Martino TA, Tata N, Ralph MR, Sole MJ, Belsham DD. Vascular circadian rhythms in a mouse vascular smooth muscle cell line (Movas-1). Am J Physiol Regul Integr Comp Physiol. 2008;295(5):R1529-R1538.

70. Xie Z, et al. Smooth-muscle BMAL1 participates in blood pressure circadian rhythm regulation. J Clin Invest. 2015;125(1):324-336.

71. Richards J, et al. A role for the circadian clock protein Per1 in the regulation of aldosterone levels and renal $\mathrm{Na}^{+}$retention. Am J Physiol Renal Physiol. 2013;305(12):F1697-F1704.

72. Gumz ML, et al. The circadian clock protein Period 1 regulates expression of the renal epithelial sodium channel in mice. J Clin Invest. 2009;119(8):2423-2434.

73. Stow $\mathrm{LR}$, et al. The circadian protein period 1 contributes to blood pressure control and coordinately regulates renal sodium transport genes. Hypertension. 2012;59(6):1151-1156.

74. Curtis AM, Cheng Y, Kapoor S, Reilly D, Price TS, Fitzgerald GA. Circadian variation of blood pressure and the vascular response to asynchronous stress. Proc Natl Acad Sci U S A. 2007;104(9):3450-3455

75. McDearmon EL, et al. Dissecting the functions of the mammalian clock protein BMAL1 by tissue-specific rescue in mice. Science. 
2006;314(5803):1304-1308.

76. Mieda M, Sakurai T. Bmal1 in the nervous system is essential for normal adaptation of circadian locomotor activity and food intake to periodic feeding. J Neurosci. 2011;31(43):15391-15396.

77. O'Brien E, Sheridan J, O'Malley K. Dippers and non-dippers. Lancet. 1988;2(8607):397.

78. Dhaun N, et al. Diurnal variation in blood pressure and arterial stiffness in chronic kidney disease: the role of endothelin-1. Hypertension. 2014;64(2):296-304.

79. Zweiker R, Eber B, Schumacher M, Toplak H, Klein W. "Non-dipping" related to cardiovascular events in essential hypertensive patients. Acta Med Austriaca. 1994;21(3):86-89.

80. Eguchi K, et al. Nocturnal nondipping of heart rate predicts cardiovascular events in hypertensive patients. JHypertens. 2009;27(11):2265-2270.

81. Aschoff J. Exogenous and endogenous components in circadian rhythms. Cold Spring Harb Symp Quant Biol. 1960;25:11-28.

82. Minors DS, Waterhouse JM. The use of constant routines in unmasking the endogenous component of human circadian rhythms. Chronobiol Int. 1984;1(3):205-216.

83. Shea SA, Hilton MF, Hu K, Scheer FA. Existence of an endogenous circadian blood pressure rhythm in humans that peaks in the evening. Circ Res. 2011;108(8):980-984.

84. Shea SA, Hilton MF, Orlova C, Ayers RT, Mantzoros CS. Independent circadian and sleep/wake regulation of adipokines and glucose in humans. JClin Endocrinol Metab. 2005;90(5):2537-2544.

85. Spengler CM, Shea SA. Endogenous circadian rhythm of pulmonary function in healthy humans. Am J Respir Crit Care Med. 2000;162(3 Pt 1):1038-1046.

86. Spengler CM, Czeisler CA, Shea SA. An endogenous circadian rhythm of respiratory control in humans. JPhysiol (Lond). 2000;526(Pt 3):683-694.

87. Zhong $X$, et al. Increased sympathetic and decreased parasympathetic cardiovascular modulation in normal humans with acute sleep deprivation. J Appl Physiol. 2005;98(6):2024-2032.

88. Lusardi P, Zoppi A, Preti P, Pesce RM, Piazza E, Fogari R. Effects of insufficient sleep on blood pressure in hypertensive patients: a 24-h study. Am J Hypertens. 1999;12(1 pt 1):63-68.

89. Butler MP, et al. The circadian system contributes to apnea lengthening across the night in obstructive sleep apnea. Sleep. 2015;38(11):1793-1801.

90. Scheer FA, et al. Impact of the human circadian system, exercise, and their interaction on cardiovascular function. Proc Natl Acad Sci U S A. 2010;107(47):20541-20546.

91. Hu K, Scheer FA, Laker M, Smales C, Shea SA. Endogenous circadian rhythm in vasovagal response to head-up tilt. Circulation. 2011;123(9):961-970.

92. Scheer FA, Hilton MF, Mantzoros CS, Shea SA. Adverse metabolic and cardiovascular consequences of circadian misalignment. Proc Natl Acad Sci U S A. 2009;106(11):4453-4458.

93. Wehrens SMT, et al. meal timing regulates the human circadian system. Curr Biol. 2017;27(12):1768-1775.e3.

94. Taylor KS, Heneghan CJ, Stevens RJ, Adams EC, Nunan D, Ward A. Heterogeneity of prognostic studies of 24-hour blood pressure variability: systematic review and meta-analysis. PLoS One. 2015;10(5):e0126375.

95. Fagard RH, Thijs L, Staessen JA, Clement DL, De Buyzere ML, De Bacquer DA. Night-day blood pressure ratio and dipping pattern as predictors of death and cardiovascular events in hypertension. J Hum Hypertens. 2009;23(10):645-653.

96. Ohkubo T, et al. Relation between nocturnal decline in blood pressure and mortality. The Ohasama Study. Am J Hypertens. 1997;10(11):1201-1207.

97. Hodgkinson J, et al. Relative effectiveness of clinic and home blood pressure monitoring compared with ambulatory blood pressure monitoring in diagnosis of hypertension: systematic review. BMJ. 2011;342:d3621.

98. Kario K, Shimada K, Pickering TG. Clinical implication of morning blood pressure surge in hypertension. J Cardiovasc Pharmacol. 2003;42(Suppl 1):S87-S91.

99. Dolan E, et al. Superiority of ambulatory over clinic blood pressure measurement in predicting mortality: the Dublin outcome study. Hypertension. 2005;46(1):156-161.

100. Kerkhof GA, Van Dongen HP, Bobbert AC. Absence of endogenous circadian rhythmicity in blood pressure? Am J Hypertens. 1998;11(3 Pt 1):373-377.

101.Van Dongen HP, Maislin G, Kerkhof GA. Repeated assessment of the endogenous 24-hour profile of blood pressure under constant routine. Chronobiol Int. 2001;18(1):85-98.

102.Zeitzer JM, Dijk DJ, Kronauer R, Brown E, Czeisler C. Sensitivity of the human circadian pacemaker to nocturnal light: melatonin phase resetting and suppression. J Physiol (Lond). 2000;526(Pt 3):695-702.

103. Gooley JJ, et al. Exposure to room light before bedtime suppresses melatonin onset and shortens melatonin duration in humans. J Clin Endocrinol Metab. 2011;96(3):E463-E472.

104.Grossman E, et al. Melatonin reduces night blood pressure in patients with nocturnal hypertension. Am JMed. 2006;119(10):898-902.

105. Burgess HJ, Trinder J, Kim Y, Luke D. Sleep and circadian influences on cardiac autonomic nervous system activity. Am J Physiol. 1997; 273(4 Pt 2):H1761-H1768.

106. Hilton MF, Umali MU, Czeisler CA, Wyatt JK, Shea SA. Endogenous circadian control of the human autonomic nervous system. Comput Cardiol. 2000;27:197-200.

107. Hu K, et al. Endogenous circadian rhythm in an index of cardiac vulnerability independent of changes in behavior. Proc Natl Acad Sci U S A. 2004;101(52):18223-18227.

108. Ho KK, et al. Predicting survival in heart failure case and control subjects by use of fully automated methods for deriving nonlinear and conventional indices of heart rate dynamics. Circulation. 1997;96(3):842-848.

109. Tofler GH, et al. Concurrent morning increase in platelet aggregability and the risk of myocardial infarction and sudden cardiac death. $N$ Engl J Med.1987;316(24):1514-1518.

110.Andreotti F, et al. Major circadian fluctuations in fibrinolytic factors and possible relevance to time of onset of myocardial infarction, sud- den cardiac death and stroke. Am J Cardiol. 1988;62(9):635-637.

111. Scheer FA, Shea SA. Human circadian system causes a morning peak in prothrombotic plasminogen activator inhibitor-1 (PAI-1) independent of the sleep/wake cycle. Blood. 2014;123(4):590-593.

112. Maemura K, et al. CLIF, a novel cycle-like factor, regulates the circadian oscillation of plasminogen activator inhibitor-1 gene expression. J Biol Chem. 2000;275(47):36847-36851.

113. Wang J, Yin L, Lazar MA. The orphan nuclear receptor Rev-erb alpha regulates circadian expression of plasminogen activator inhibitor type 1. J Biol Chem. 2006;281(45):33842-33848.

114. Ohkura N, et al. Circadian clock molecules CLOCK and CRYs modulate fibrinolytic activity by regulating the PAI-1 gene expression. J Thromb Haemost. 2006;4(11):2478-2485.

115. Scheer FA, et al. The human endogenous circadian system causes greatest platelet activation during the biological morning independent of behaviors. PLoS One. 2011;6(9):e24549.

116. Ridker PM, Manson JE, Buring JE, Muller JE, Hennekens $\mathrm{CH}$. Circadian variation of acute myocardial infarction and the effect of low-dose aspirin in a randomized trial of physicians. Circulation. 1990;82(3):897-902.

117. Moncada S, Palmer RM, Higgs EA. The discovery of nitric oxide as the endogenous nitrovasodilator. Hypertension. 1988;12(4):365-372.

118. Palmer RM, Ferrige AG, Moncada S. Nitric oxide release accounts for the biological activity of endothelium-derived relaxing factor. Nature. 1987;327(6122):524-526.

119. Radomski MW, Palmer RM, Moncada S. Endogenous nitric oxide inhibits human platelet adhesion to vascular endothelium. Lancet. 1987;2(8567):1057-1058.

120. Halcox JP, et al. Prognostic value of coronary vascular endothelial dysfunction. Circulation. 2002;106(6):653-658.

121. Viswambharan $\mathrm{H}$, et al. Mutation of the circadian clock gene Per2 alters vascular endothelial function. Circulation. 2007;115(16):2188-2195.

122.Wang CY, et al. Increased vascular senescence and impaired endothelial progenitor cell function mediated by mutation of circadian gene Per2. Circulation. 2008;118(21):2166-2173.

123. Jones H, Green DJ, George K, Atkinson G. Intermittent exercise abolishes the diurnal variation in endothelial-dependent flow-mediated dilation in humans. Am J Physiol Regul Integr Comp Physiol. 2010;298(2):R427-R432.

124. Elherik K, Khan F, McLaren M, Kennedy G, Belch $\mathrm{JJ}$. Circadian variation in vascular tone and endothelial cell function in normal males. Clin Sci. 2002;102(5):547-552.

125. Kawano H, et al. Endothelial function fluctuates with diurnal variation in the frequency of ischemic episodes in patients with variant angina. JAm Coll Cardiol. 2002;40(2):266-270.

126. Gaenzer H, Sturm W, Kirchmair R, Neumayr G, Ritsch A, Patsch J. Circadian variation of endothelium-dependent vasodilatation of the brachial artery as a confounding factor in the evaluation of endothelial function. Atherosclerosis. 2000;149(1):227-228. 
127. Etsuda H, et al. Morning attenuation of endothelium-dependent, flow-mediated dilation in healthy young men: possible connection to morning peak of cardiac events? Clin Cardiol. 1999;22(6):417-421.

128. Maruo T, et al. Circadian variation of endothelial function in idiopathic dilated cardiomyopathy. Am J Cardiol. 2006;97(5):699-702.

129. Jones H, Lewis NC, Thompson A, Marrin K, Green DJ, Atkinson G. Diurnal variation in vascular function: role of sleep. Chronobiol Int. 2012;29(3):271-277.

130. Willich SN, Lewis M, Löwel H, Arntz HR, Schubert F, Schröder R. Physical exertion as a trigger of acute myocardial infarction. Triggers and Mechanisms of Myocardial Infarction Study Group. N Engl J Med. 1993;329(23):1684-1690.

131. Witte K, Parsa-Parsi R, Vobig M, Lemmer B. Mechanisms of the circadian regulation of beta-adrenoceptor density and adenylyl cyclase activity in cardiac tissue from normotensive and spontaneously hypertensive rats. J Mol Cell Cardiol. 1995;27(5):1195-1202.

132. Hughes ME, et al. Harmonics of circadian gene transcription in mammals. PLoS Genet. 2009;5(4):e1000442.

133. Knutsson A, Jonsson B, Akerstedt T, Orth-Gomer $\mathrm{K}$. Increased risk of ischaemic heart disease in shift workers. Lancet. 1986;2(8498):89-92.

134. Karlsson B, Knutsson A, Lindahl B. Is there an association between shift work and having a metabolic syndrome? Results from a population based study of 27,485 people. Occup Environ Med. 2001;58(11):747-752.

135. Gu F, et al. Total and cause-specific mortality of U.S. nurses working rotating night shifts. $A m$ Prev Med. 2015;48(3):241-252.

136. Wegrzyn LR, et al. Rotating night-shift work and the risk of breast cancer in the nurses' health studies. Am J Epidemiol. 2017;186(5):532-540.

137. Buxton OM, et al. Adverse metabolic consequences in humans of prolonged sleep restriction combined with circadian disruption. Sci Transl Med. 2012;4(129):129ra43.

138. Buxton OM, Pavlova M, Reid EW, Wang W, Simonson DC, Adler GK. Sleep restriction for 1 week reduces insulin sensitivity in healthy men. Diabetes. 2010;59(9):2126-2133.

139. Spiegel K, Knutson K, Leproult R, Tasali E, Van Cauter E. Sleep loss: a novel risk factor for insulin resistance and Type 2 diabetes. J Appl Physiol. 2005;99(5):2008-2019.

140. Leproult R, Holmbäck U, Van Cauter E. Circadian misalignment augments markers of insulin resistance and inflammation, independently of sleep loss. Diabetes. 2014;63(6):1860-1869.

141. Meigs JB, Nathan DM, D’Agostino RB, Wilson PW, Framingham Offspring Study. Fasting and postchallenge glycemia and cardiovascular disease risk: the Framingham Offspring Study. Diabetes Care. 2002;25(10):1845-1850.

142. Morris CJ, Purvis TE, Hu K, Scheer FA. Circadian misalignment increases cardiovascular disease risk factors in humans. Proc Natl Acad Sci U S A. 2016;113(10):E1402-E1411.

143. Penev PD, Kolker DE, Zee PC, Turek FW. Chronic circadian desynchronization decreases the survival of animals with cardiomyopathic heart disease.
Am J Physiol. 1998;275(6 Pt 2):H2334-H2337.

144. Hurd MW, Ralph MR. The significance of circadian organization for longevity in the golden hamster. J Biol Rhythms. 1998;13(5):430-436.

145. Alibhai FJ, et al. Short-term disruption of diurnal rhythms after murine myocardial infarction adversely affects long-term myocardial structure and function. Circ Res. 2014;114(11):1713-1722.

146.Eckle T, et al. Adora2b-elicited Per2 stabilization promotes a HIF-dependent metabolic switch crucial for myocardial adaptation to ischemia. Nat Med. 2012;18(5):774-782.

147. Durgan DJ, et al. Short communication: ischemia/reperfusion tolerance is time-of-daydependent: mediation by the cardiomyocyte circadian clock. Circ Res. 2010;106(3):546-550.

148. Boivin DB, Boudreau P, James FO, Kin NM. Photic resetting in night-shift work: impact on nurses' sleep. Chronobiol Int. 2012;29(5):619-628.

149. Eastman CI, Stewart KT, Mahoney MP, Liu L, Fogg LF. Dark goggles and bright light improve circadian rhythm adaptation to night-shift work. Sleep. 1994;17(6):535-543.

150. Crowley SJ, Lee C, Tseng CY, Fogg LF, Eastman CI. Combinations of bright light, scheduled dark, sunglasses, and melatonin to facilitate circadian entrainment to night shift work. J Biol Rhythms. 2003;18(6):513-523.

151. Boudreau P, Dumont GA, Boivin DB. Circadian adaptation to night shift work influences sleep, performance, mood and the autonomic modulation of the heart. PLoS One. 2013;8(7):e70813.

152. Akerstedt T. Shift work and disturbed sleep/wakefulness. Occup Med (Lond). 2003;53(2):89-94.

153. Wittmann M, Dinich J, Merrow M, Roenneberg T. Social jetlag: misalignment of biological and social time. Chronobiol Int. 2006;23(1-2):497-509.

154. Roenneberg T, Allebrandt KV, Merrow M, Vetter C. Social jetlag and obesity. Curr Biol. 2012;22(10):939-943.

155. Rutters F, et al. Is social jetlag associated with an adverse endocrine, behavioral, and cardiovascular risk profile? J Biol Rhythms. 2014;29(5):377-383.

156. Parsons MJ, et al. Social jetlag, obesity and metabolic disorder: investigation in a cohort study. Int JObes (Lond). 2015;39(5):842-848.

157. Jiddou MR, Pica M, Boura J, Qu L, Franklin BA. Incidence of myocardial infarction with shifts to and from daylight savings time. Am J Cardiol. 2013;111(5):631-635.

158. Smith MR, Eastman CI. Shift work: health, performance and safety problems, traditional countermeasures, and innovative management strategies to reduce circadian misalignment. Nat Sci Sleep. 2012;4:111-132.

159. Yamaguchi $Y$, et al. Mice genetically deficient in vasopressin $\mathrm{V} 1 \mathrm{a}$ and $\mathrm{V} 1 \mathrm{~b}$ receptors are resistant to jet lag. Science. 2013;342(6154):85-90.

160. Mensah GA, Mokdad AH, Ford ES, Greenlund $\mathrm{KJ}$, Croft JB. State of disparities in cardiovascular health in the United States. Circulation. 2005;111(10):1233-1241.

161. Smith MR, Burgess HJ, Fogg LF, Eastman CI. Racial differences in the human endogenous circadian period. PLoS One. 2009;4(6):e6014.

162. Hirota T, et al. Identification of small molecule activators of cryptochrome. Science. 2012;337(6098):1094-1097.
163. He B, et al. The small molecule nobiletin targets the molecular oscillator to enhance circadian rhythms and protect against metabolic syndrome. Cell Metab. 2016;23(4):610-621.

164. Meng QJ, et al. Ligand modulation of REVERBalpha function resets the peripheral circadian clock in a phasic manner. J Cell Sci. 2008;121(pt 21):3629-3635.

165. Solt LA, et al. Regulation of circadian behaviour and metabolism by synthetic REV-ERB agonists. Nature. 2012;485(7396):62-68.

166. Woldt E, et al. Rev-erb- $\alpha$ modulates skeletal muscle oxidative capacity by regulating mitochondrial biogenesis and autophagy. Nat Med 2013;19(8):1039-1046

167. Montaigne D, et al. Daytime variation of perioperative myocardial injury in cardiac surgery and its prevention by Rev-Erb $\alpha$ antagonism: a singlecentre propensity-matched cohort study and a randomised study. Lancet. 2018;391(10115):59-69.

168. Birkenhäger AM, van den Meiracker AH. Cause and consequences of a non-dipping blood pressure profile. Neth J Med. 2007;65(4):127-131.

169. Munger MA, Van Tassell BW, LaFleur J. Medication nonadherence: an unrecognized cardiovascular risk factor. MedGenMed. 2007;9(3):58.

170.Pincus DJ, Szefler SJ, Ackerson LM, Martin RJ. Chronotherapy of asthma with inhaled steroids: the effect of dosage timing on drug efficacy. J Allergy Clin Immunol. 1995;95(6):1172-1178.

171. Zang XY, Liu JF, Chai YF, Wong FK, Zhao Y. Effect on blood pressure of a continued nursing intervention using chronotherapeutics for adult Chinese hypertensive patients. J Clin Nurs. 2010;19(7-8):1149-1156.

172. Levi F, Schibler U. Circadian rhythms: mechanisms and therapeutic implications. Annu Rev Pharmacol Toxicol. 2007;47:593-628.

173. Litinski M, Scheer FA, Shea SA. Influence of the circadian system on disease severity. Sleep Med Clin. 2009;4(2):143-163.

174. Cohn PF, Lawson WE. Effects of long-acting propranolol on A.M. and P.M. peaks in silent myocardial ischemia. Am J Cardiol. 1989;63(12):872-873.

175. Sica D, Frishman WH, Manowitz N. Pharmacokinetics of propranolol after single and multiple dosing with sustained release propranolol or propranolol CR (innopran XL), a new chronotherapeutic formulation. Heart Dis. 2003;5(3):176-181.

176. Kuroda T, et al. Effects of bedtime vs. morning administration of the long-acting lipophilic angiotensin-converting enzyme inhibitor trandolapril on morning blood pressure in hypertensive patients. Hypertens Res. 2004;27(1):15-20.

177. Bem D, et al. The effects of different aspirin dosing frequencies and the timing of aspirin intake in primary and secondary prevention of cardiovascular disease: a systematic review. Clin Pharmacol Ther. 2016;100(5):500-512.

178. Hermida RC, Ayala DE, Calvo C, López JE. Aspirin administered at bedtime, but not on awakening, has an effect on ambulatory blood pressure in hypertensive patients. J Am Coll Cardiol. 2005;46(6):975-983.

179. Martino TA, et al. The primary benefits of angiotensin-converting enzyme inhibition on cardiac remodeling occur during sleep time in murine pressure overload hypertrophy. J Am Coll Cardiol. 
2011;57(20):2020-2028.

180.Hermida RC, Ayala DE, Mojón A, Fernández JR. Influence of circadian time of hypertension treatment on cardiovascular risk: results of the MAPEC study. Chronobiol Int. 2010;27(8):1629-1651.

181. Hermida RC, Ayala DE, Mojón A, Fernández JR. Influence of time of day of blood pressure-lowering treatment on cardiovascular risk in hyperten- sive patients with type 2 diabetes. Diabetes Care. 2011;34(6):1270-1276.

182. Scheer FA, Stone PH, Shea SA. Decreased sleep in heart failure: are medications to blame? Arch Intern Med. 2007;167(10):1098-1099; author reply 1099.

183. Kario K, et al. Morning surge in blood pressure as a predictor of silent and clinical cerebrovascular disease in elderly hypertensives: a prospective study. Circulation. 2003;107(10):1401-1406.

184.Rana JS, Mukamal KJ, Morgan JP, Muller JE, Mittleman MA. Circadian variation in the onset of myocardial infarction: effect of duration of diabetes. Diabetes. 2003;52(6):1464-1468.

185. Gami AS, Howard DE, Olson EJ, Somers VK. Daynight pattern of sudden death in obstructive sleep apnea. N Engl J Med. 2005;352(12):1206-1214. 